\title{
IMPROVED TURNOVER PREDICTION OF SHARES USING HYBRID FEATURE SELECTION
}

\author{
Shashaank D.S, Sruthi.V, Vijayalashimi M.L.S and Shomona Garcia Jacob \\ Department of Computer Science and Engineering, \\ SSNCE, Chennai, India
}

\begin{abstract}
Predicting the total turnover of a company in the most unstable stock market and trade conditions has always proved to be a costly affair causing rise and fall of several trades. Data mining is a well-known sphere of Computer Science that aims at extracting meaningful information from large databases. However, despite the existence of many algorithms for the purpose of predicting future trends, their efficiency is questionable as their predictions suffer from a high error rate. The objective of this paper is to investigate and rate the performance of classifiers based on the features selected by Hybrid Feature Selection. The authorized dataset for predicting the turnover was taken fromwww.bsc.com and included the stock market values of various companies over the past 10 years. The algorithms were investigated using the Weka tool. The Hybrid feature selection (HFS) algorithm, was run on this dataset to extract the important and influential features for classification. With these extracted features, the Total Turnover of the company was predicted using various algorithms like Random Forest, Decision Tree, SVM and Multinomial Regression. This prediction mechanism was implemented to predict the turnover of a company on an everyday basis and hence could help navigate through dubious stock markets trades. An accuracy rate of was achieved by the above prediction process. Moreover, the importance of the stock market attributes through Incremental Feature Selection (IFS) was established as well.
\end{abstract}

\section{KEYWORDS}

Data mining, Hybrid Feature selection, classification algorithms, Turnover prediction

\section{INTRODUCTION}

Prediction of stock market prices, its rise and fall of values has constantly proved to be a perilous task mainly due to the volatile nature of the market[1-3]. However data mining techniques and other computational intelligence techniques have been applied to achieve the same over the years. Some of the approaches undertaken included the use of decision tree algorithm, concepts of neural networks and Midas[4-6]. However through this paper, a comparative study was conducted to estimate and predict the turnover of companies that include Infosys, Sintex, HDFC and Apollo hospitals using the features selected by the Hybrid Feature Selection algorithm (Ramani et al, 2013) by utilizing classifiers such as Naive Bayes, Bayesian Networks, Random Forest, Nearest Neighor and J48. In order to estimate the performance of the aforementioned machine learning algorithms in predicting the turnover, a confusion matrix was also constructed with respect to the dataset. Based on the predictions made by each of the algorithms with respect 
to the total turnover for a company (on an everyday basis), an accuracy rate was estimated for each of them from the number of true positives/negatives and false positives/negatives. A brief review of the state-of-the-art in predicting stock market share data is given below.

\section{RELATED WORK}

The objective of any nation at large is to enhance the lifestyle of common man and that is the driving force to undertake research to predict the market trends [7-9]. In the recent decade, much research has been done on neural networks to predict the stock market changes [10].

Matsui and Sato [12] proposed a new evaluation method to dissolve the over fitting problem in the Genetic Algorithm (GA) training. On comparing the conventional and the neighbourhood evaluation they found the new evaluation method to be better than the conventional one in terms of performance. Gupta, Aditya, and Dhingra [13] proposed a stock market prediction technique based on Hidden Markov Models. In that approach, the authors considered the fractional change in stock value and the intra-day high and low values of the stock to train the continuous Hidden Markov Model (HMM).Then this HMM is used to make a Maximum a Posteriori decision over all the possible stock values for the next day. The authors applied this approach on several stocks, and compared the performance to the existing methods. Lin, Guo, and $\mathrm{Hu}[14]$ proposed a SVM based stock market prediction system .This system selected a good feature subset, evaluated stock indicator and controlled over fitting on stock market tendency prediction. The authors tested this approach on Taiwan stock market datasets and found that the proposed system surpassed the conventional stock market prediction system in terms of performance. Ramani et al(2013) proposed the Hybrid Feature Selection methodology on Clinical Lung Cancer datasets wherein the authors concluded that combining the gain ratio with correlation of features to each other and to the target class resulted in an optimal feature subset. This was also followed by Incremental Feature Selection as discussed in the ensuing section.

\section{PROPOSED HFS BASED STOCK TURNOVER PREDICTION FRAMEWORK}

The stock turnover prediction framework proposed in this paper is portrayed in Figure 1. The basic methodology involved Data Collection, Pre-processing, Hybrid Feature Selection and Classification, each of which is explained below.

The dataset utilised for predicting the turnover was taken from www.bsc.com which included the stock market values of companies including Infosys, HDFC, Apollo Hospitals and Sintex, over the past 10 years. 


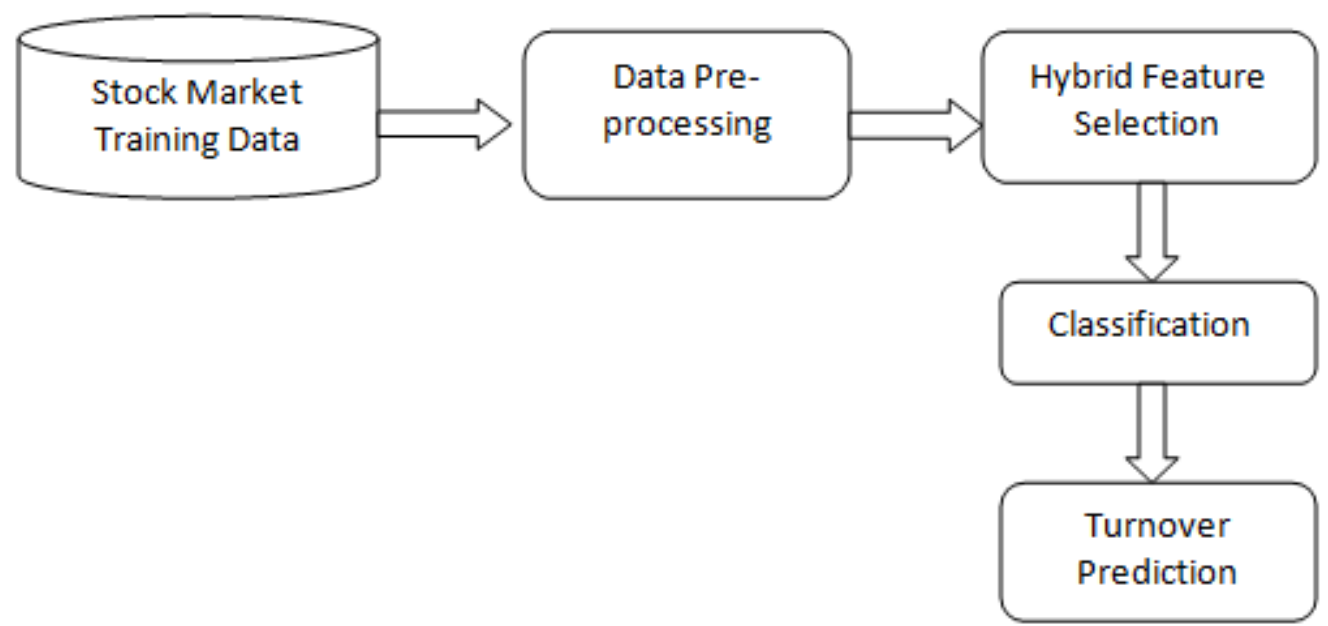

Figure 1: Stock Turnover Prediction framework through HFS

\subsection{Data Processing}

Initially, all the records with missing values were removed from the dataset in order to improve the accuracy of the prediction. Then the data was further partitioned into two parts:

Training data (d.t): It is the data with which the machine is trained. Various classification algorithms are trained on this data. $60 \%$ of the data is taken as training data.

Validation data (d.v):It is the data which is used for the purpose of cross-validation. It is used to find the accuracy rate of each algorithm. The remaining $40 \%$ of the data is taken as validation data.

In order to apply the classification algorithms, the data was first sorted according to the turnover. Then the total turnover was discretised into:

$$
\begin{aligned}
& \text { A - 58,320 to } 18,291,986 \\
& \text { B - } 18,296,597 \text { to } 37,731,606 \\
& \text { C }-37,749,751 \text { to } 121,233,543 \\
& \text { D }-121,245,870 \text { to } 300,360,881 \\
& \text { E- } 300,465,316 \text { to } 19,085,311,470
\end{aligned}
$$

Also, the company features were converted into dummy variables $(0$ 's $/ 1$ 's) to help the prediction process easier.

The stock market data was characterized by attributes described in Table 1 . The stock market starts at 9:15 in the morning and ends at 3:30 in the afternoon. The attributes described in Table 1 are recorded within this time frame. 
International Journal of Data Mining \& Knowledge Management Process (IJDKP) Vol.5, No.6, November 2015

Table 1. Stock Market Share Data - Attribute Description

\begin{tabular}{|c|c|c|}
\hline S.NO & ATTRIBUTE & DESCRIPTION \\
\hline 1. & Open price & $\begin{array}{l}\text { The first traded price during the day or in the } \\
\text { morning. }\end{array}$ \\
\hline 2. & High price & The highest traded price during the day. \\
\hline 3. & Low price & The lowest price traded during the day. \\
\hline 4. & Close price & The last price traded during the day. \\
\hline 5. & WAP & Weighted average price during the day. \\
\hline 6. & No of shares & $\begin{array}{l}\text { The total number of shares done during the } \\
\text { day. }\end{array}$ \\
\hline 7. & No of trades & $\begin{array}{l}\text { No of trades is the total no of transactions } \\
\text { during the day. }\end{array}$ \\
\hline 8. & $\begin{array}{l}\text { Deliverable } \\
\text { quantity. }\end{array}$ & $\begin{array}{l}\text { The quantity that can be delivered at the end of } \\
\text { the day. }\end{array}$ \\
\hline 9. & Spread high low & Range of High price and low prices. \\
\hline 10. & Spread close open & Range of close and low prices. \\
\hline 11. & Company & $\begin{array}{l}\text { The name of the company that handles the } \\
\text { shares. }\end{array}$ \\
\hline 12. & Total turn over & $\begin{array}{l}\text { Turnover is the total no of shares traded } \mathrm{X} \\
\text { Price of each share sold. }\end{array}$ \\
\hline 13. & Date & $\begin{array}{l}\text { The date for which the above attributes are } \\
\text { recorded. }\end{array}$ \\
\hline
\end{tabular}

Once the data was pre-processed, the important features to make an accurate prediction were identified by the process of feature selection.

\subsection{Feature Selection}

Feature ranking presented significant features in the order of their contribution to categorizing the samples under the different target classes. Since most feature selection algorithms focused on ranking the attributes according to their significance value, the liability of choosing the limiting constraint rested with the user. Hence in order to automate the process of finding the minimal yet optimal set of features, the ranking feature selection algorithms were followed by Correlation Subset Evaluators that included features highly correlated to the class and least correlated to each other. Since both the ranking and subset evaluators were utilized to obtain the optimal feature set, 
this was termed the Hybrid Feature Selection strategy. The description of the methods used in this research is detailed below.

Gain ratio criterion [19]revealed the association between an attribute and the class value, being primarily computed from the Information Gain using the Information Entropy (InfoE) values. The CFS hypothesis suggested that the most predictive features needed to be highly correlated to the target class and least relevant to other predictor attributes. The predictor attributes generated by the Gain Ratio and CFS Subset Attribute Evaluator (Hybrid Feature Selection) methods were later utilized for Incremental Feature Selection (IFS) to determine the minimal and optimal set of features. On adding each feature, a new feature set was obtained.

\subsection{Classification}

Classification [16-19] is the process of finding a set of models that describe and distinguish data classes. This is done to achieve the goal of being able to use the model to predict the class whose label is unknown. The classification phase involved the execution of the classification algorithms to identify the best performing algorithm. The classification accuracy obtained by percentage split as discussed in the data pre-processing phase, was calculated and a comparison was drawn among the classifiers. The algorithms that yielded the highest accuracy is described below.

\section{Random Forest}

In random forest [18-19] a randomly selected set of attributes is used to split each node. Every node is split using the best split among a subset of predictors that are deliberately chosen randomly at the node. This is in contrast to the methodology followed in standard tress in which each node is split using the best split among all attributes available in the dataset. Further new values are predicted by aggregating and collating the predictions of the various decision trees constructed.

Random forest represents an ensemble model / algorithm as it derives its final prediction from multiple individual models. These individual models could be of similar or different type. However, in the case of Random Forest, the individual models are of the same type - decision trees.

The Random Forest algorithm yielded 97.2\% accuracy with the three features, No.of shares, No.of trades and Close_price.

The performances of the feature selection and classification algorithms are discussed below.

\section{RESULT ANALYSIS}

The results analysis is discussed in two sections. The former section elaborates on the feature selection process while the latter section makes a detailed analysis on the performance of the classification algorithms.

\subsection{Performance Analysis of Feature Selection}

The Hybrid Feature Selection algorithm resulted in an optimal feature subset that contained only 3 features viz, No. of shares, No. of trades and closing price. The Gain Ratio of these features is depicted in Table 2. 
International Journal of Data Mining \& Knowledge Management Process (IJDKP) Vol.5, No.6, November 2015

Table 2. Attribute Importance in Turnover Prediction - Gain Ratio Ranking

\begin{tabular}{|l|l|l|}
\hline S1.No & Feature Selected & Gain Ratio \\
\hline 1. & No.of Shares & 0.252 \\
\hline 2. & No. of trades & 0.2349 \\
\hline 3. & Closing _ Price & 0.2212 \\
\hline
\end{tabular}

Once the important features were identified, the next phase involved predicting the turnover from the features in order to estimate the probable combination of attributes that yield a high turnover.

\subsection{Performance Analysis of Classification Algorithms}

Each of the classification algorithms were first trained using the training data which contained $60 \%$ of the dataset. The 10-fold cross-validation method was employed to evaluate the performance of the classification algorithms and the obtained accuracy is depicted in Table 3.

Accuracy rate $=\underline{\text { No. of correctly classified observations }} \times 100$

Total No. of observations

Table 3. Comparative Performance of Classification Algorithms

\begin{tabular}{|c|c|c|}
\hline S.No & Classification Algorithms & Accuracy (\%) \\
\hline 1 & Random Forest & 97.2 \\
\hline 2 & J48 & 95.7 \\
\hline 3 & Nearest-Neighbour & 81.8 \\
\hline 4 & Bayesian Network & 73.7 \\
\hline 5 & Naive Bayes & 57.5 \\
\hline
\end{tabular}

The graphical representation of the total turnover prediction of the companies is given in Figure 2 .

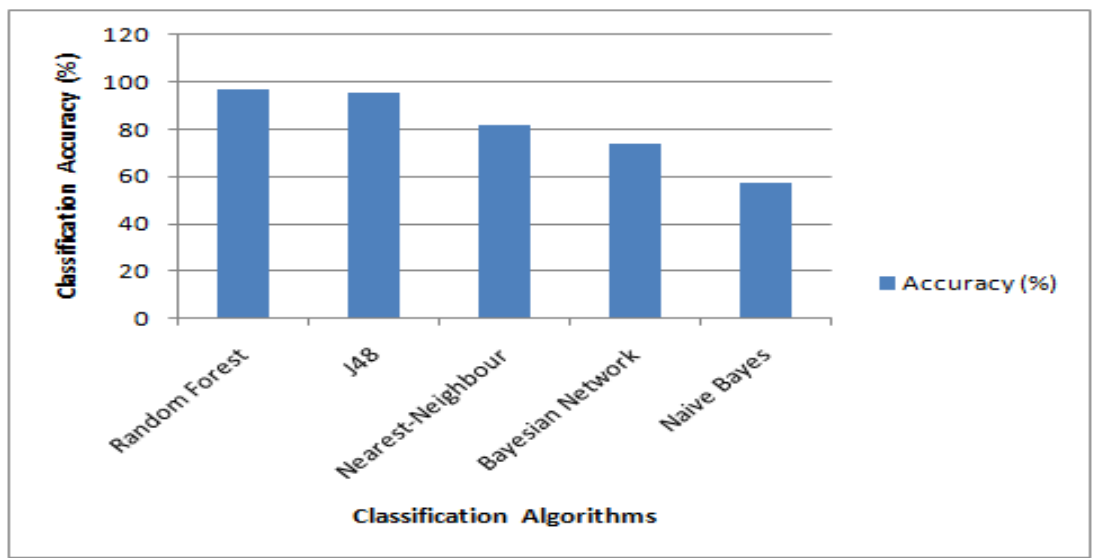

Figure 2 - Comparative Performance of Classification Algorithms

It is evident from the result analysis that a small subset of features is sufficient to accurately predict the total turnover of a company. Moreover the Random Forest algorithm has proved to 
accurately predict the turnover for the real-time share data of different companies which gives the lead to investigate many other boosting and ensemble techniques to enhance the prediction accuracy.

\section{CONCLUSION}

Application of data mining techniques to predict turnover based on stock market share data is an emerging area of research and is expected to be instrumental in moulding the country's economy by predicting possible investment trends to increase turnover. In view of this, an efficient way of implementing the Random Forest algorithm with Hybrid Feature Selection is proposed in order to mitigate the risks involved in predicting the turnover of a company. The optimal feature subset was also identified to predict the turnover with maximum accuracy. An accuracy rate of $97 \%$ was achieved in the prediction process. This accuracy rate was much higher than those obtained before. Hence we believe that further research using computational methodologies to predict turnover on a daily basis based on share market data will reveal better and more interesting patters for investments.

\section{REFERENCES}

[1] Abhishek Gupta, Dr. Samidha , D. Sharma - "Clustering-Classification Based Prediction of Stock Market Future Prediction" - (IJCSIT) International Journal of Computer Science and Information Technologies, Vol. 5 (3) , 2014, 2806-2809.

[2] Dharamveer, Beerendra , Jitendra Kumar -“ Efficient Prediction of Close Value using Genetic algorithm based horizontal partition decision tree in Stock Market “ Volume 2, Issue 1, January 2014 International Journal of Advance Research in Computer Science and Management Studies Research Paper Available online at: www.ijarcsms.com.

[3] Kannan, K. Senthamarai, et al. "Financial stock market forecast using data mining techniques." Proceedings of the International Multiconference of Engineers and computer scientists. Vol. 1. 2010. [4] Md. Al Mehedi Hasan, Mohammed Nasser, Biprodip Pal, Shamim Ahmad - "Support Vector Machine and Random Forest Modeling for Intrusion Detection System (IDS)"- Journal of Intelligent Learning Systems and Applications, Vol.6 No.1(2014), Article ID:42869,8 pages

[4] Shen, S., Jiang, H., \& Zhang, T. (2012). Stock market forecasting using machine learning algorithms.

[5] Bayaga, Anass. "Multinomial logistic regression: usage and application in risk analysis." Journal of applied quantitative methods 5.2 (2010): 288-297.

[6] Shah, Vatsal H. "Machine learning techniques for stock prediction." Foundations of Machine Learningl Spring (2007).

[7] Al-Radaideh, Qasem A., Aa Assaf, And Eman Alnagi. "Predicting Stock Prices Using Data Mining Techniques." The International Arab Conference on Information Technology (ACIT’2013). 2013.

[8] Wang, Jar-Long, and Shu-Hui Chan. "Stock market trading ruleDiscovery using two-layer bias decision tree." Expert Systems withApplications 30.4, 605-611, 2006.

[9] Wu, Muh-Cherng, Sheng-Yu Lin, and Chia-Hsin Lin. "An effectiveApplication of decision tree to stock trading." Expert Systems withApplications 31.2: 270-274, 2006.

[10] Mahdi Pakdaman Naeini, Hamidreza Taremian, Homa Baradaran Hashemi "Stock Market Value Prediction Using Neural Networks", International Conference on Computer Information Systems and Industrial Management Applications (CISIM), pp. 132-136, 2010.

[11] Matsui, Kazuhiro, and Haruo Sato. "Neighbourhood evaluation in acquiring stock trading strategy using genetic algorithms." Soft Computing and Pattern Recognition (SoCPaR), 2010 International Conference of. IEEE, 2010.

[12] Gupta, Aditya, and Bhuwan Dhingra. "Stock market prediction using hidden markov models." Engineering and Systems (SCES) Students Conference on, IEEE, 2012. 
International Journal of Data Mining \& Knowledge Management Process (IJDKP) Vol.5, No.6, November 2015

[13] Lin, Yuling, Haixiang Guo, and Jinglu Hu,"An SVM-based approach for stock market trend prediction." Neural Networks (IJCNN), the 2013 International Joint Conference on. IEEE, 2013.

[14] Sanjana Sahayaraj, Shomona Gracia Jacob, Data Mining to Help Aphasic Quadriplegic and Coma Patients, International Journal of Science and Research (IJSR), Vol. 3(9), pp.121-125, 2014.

[15] Jacob SG, Ramani RG, Prediction of Rescue Mutants to predict Functional Activity of Tumor Protein TP53 through Data Mining Technniques”, Journal of Scientific and Industrial Research, Vol.74, pp.135-140, 2015.

[16] Zhao, Yanchang. R and data mining: Examples and case studies. Academic Press, 2012.

[17] Ramani, R. Geetha, and Shomona Gracia Jacob. "Improved classification of lung cancer tumors Based on structural and physicochemical properties of proteins using data mining models." (2013): e58772.

[18] Jacob, S. G., and R. G. Ramani. "Prediction of Rescue Mutants to Restore Functional Activity of Tumor Protein TP53 through Data Mining Techniques." Journal of Scientific \& Industrial Research 74.3 (2015): 135-140.

\section{AUTHORS}

Shashaank D.S is currently pursuing B.E computer Science and Engineering in SSN College of Engineering Chennai, India. He is doing research in the field of machine learning and is interested in speech processing.

Sruthi.Vis currently pursuing B.E computer Science and Engineering in SSN College of Engineering Chennai, India. She is doing research in the field of machine learning.

Vijayalakshimi M.L.S is currently pursuing B.E computer Science and Engineering in SSN College of Engineering Chennai, India. She is doing research in the field of machine learning.

Dr. Shomona Gracia Jacob is Associate Professor, Department of CSE, SSN College of Engineering, Chennai, India. She completed Ph.D at Anna University in the area of Biological and Clinical Data Mining. She has more than 30 publications in International Conferences and Journals to her credit. Her areas of interest include Data Mining, Bioinformatics, Machine Learning, and Artificial Intelligence .She has reviewed many research articles on invitation from highly reputed refereed journals. She is currently guiding under-graduate and post-graduate projects in the field of data
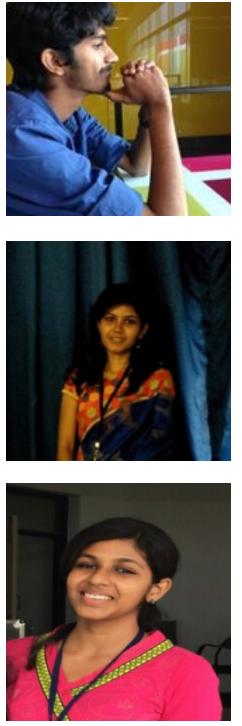
mining and intelligent systems.

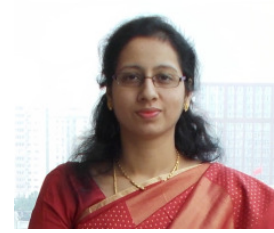

